\title{
Synthetic Bile Acids: Novel Mediators of Apoptosis
}

\author{
Nam Deuk Kim*, Eun Ok Im, Yung Hyun Choi ${ }^{\dagger}$ and Young Hyun Yoo \\ Department of Pharmacy, Pusan National University, Pusan Cancer Research Center, Pusan 609-735 \\ 'Department of Biochemistry, College of Oriental Medicine, Dong-Eui University, \\ and Research Center for Oriental Medicine, Pusan 614-052, \\ Department of Anatomy and Cell Biology, \\ Dong-A University College of Medicine and Institute of Medical Science, Pusan 602-714, Korea
}

Received 15 November 2001

Bile acids are polar derivatives of cholesterol that are essential for the absorption of dietary lipids, and regulate the transcription of genes that control cholesterol homeostasis. Depending on the nature of the chemical structures, different bile acids exhibit distinct biological effects (Martinez et al., 1998). After synthesis by the liver and excretion into the bile canaliculus and the digestive tract, the primary bile acids, cholic acid (CA) and chenodeoxycholic acid (CDCA), are metabolized by enteric bacteria to produce secondary bile acids-primarily deoxycholic acid (DCA), ursodeoxycholic acid (UDCA), and lithochlic acid (LCA). Bile acids are conjugated to glycine, or taurine when the glycine conjugates predominate (Hofmann, 1984). Conjugation of bile acids to glycine and taurine is one mechanism by which an organism can decrease the hydrophobicity of a bile acid (Martinez-Diez et al., 2000; Rust et al., 2000). The conjugation renders the molecules less cytotoxic at physiological concentrations (Patel et al., 1994). Numerous studies have shown that elevated concentrations of bile acid within the liver induce hepatocyte apoptosis. This provides a cellular mechanism for bile acidmediated liver injury (Patel et al., 1998). Bile acid hydrophobicity is correlated with induction of apoptosis and/ or growth arrest (Powell et al., 2001). Highly hydrophobic bile acids, such as DCA and CDCA, are able to induce apoptosis rapidly.

Recently, it was reported that several synthetic bile acids induced apoptosis in human hepatocellular carcinoma cells, human breast carcinoma cells, and human leukemic T cells (Baek et al., 1997; Park et al., 1997; Im et al., 1999; Choi et al., 2001; Im et al., 2001), and inhibited angiogenesis in human hepatocellular carcinoma cells (Suh et al., 1997). Moreover, UDCA and its synthetic derivatives, HS-1030 and

*To whom correspondence should be addressed.

Tel: 82-51-5102801; Fax: 82-51-5136754

E-mail: nadkim@pusan.ac.kr
HS-1183, inhibited SV40 DNA replication, and predominantly inhibited the initiation stage of DNA replication (Kim et al., 1999).

In this review, we will describe the biological activity of natural bile acids and synthetic derivatives, focusing on their role as mediators of apoptosis. A discussion of the biochemical and cellular effects of synthetic bile acids will follow an introduction to the induction of apoptosis by natural bile acids. We will then discuss inducers, targets, and the mechanism of action of natural and synthetic bile acids. The chapter will conclude with a discussion on the implications of the results, and a discussion on the potential therapeutic possibilities that arise from modulation of the apoptotic process.

\section{Natural bile acids as inducers of apoptosis}

Natural bile salt-induced apoptosis is a tissue- and diseasespecific process (Blake et al., 1998). Hepatocytes, cholangiocytes, and ileal enterocytes are the only cells in the human body that can transport natural bile salts intracellularly. Different natural bile acids exhibit distinct biological effects in vivo and in vitro. Recent studies have shown that bile acids can affect intracellular signaling and gene expression, which may ultimately lead to alterations in cell growth and tumorigenesis (Qiao et al., 2001a). Several growth regulatory genes, such as cyclooxygenase-2 (Zhang et al., 1998), GADD153 (Zheng et al., 1996) and c-myc (Takai et al., 1986; Porsch Hallstrom et al., 1991), as well as transcription factors such as activator protein-1 (AP-1) (Qiao et al., 2000) and NFкB (Payne et al., 1998), are activated by bile acids.

Primary bile acid, hydrophobic CA, has no discernible effect on the human colon cancer cells, and little or no activity is provided if it is not metabolized to DCA (Martinez et al., 1998). In hepatic stellate cells, CA induces egr and fos, early response genes in the requirement of protein kinase $\mathrm{C}$ (PKC) 
$\alpha$ and/or PKC $\delta$. PKC is also known as the only cytoplasmic signaling molecule that responds to bile acids (Brady et al., 1996). Taurocholic acid is a water-soluble major secondary CA. It can activate mitogen-activated protein kinase (MAPK) in hepatic stellate cells (Brady et al., 1996). Bile acids also activate the major cytoplasmic signaling kinases of the stellate cells, which seem to regulate many plasma-membrane receptor-driven cascades that are associated with tyrosine kinase, or GTP-binding protein receptors (Hunter, 1995; Marshall, 1995; Brady et al., 1996).

CDCA, a primary bile acid, acts as a tumor promoter in animal models, enhances cell transformation in vitro, and induces apoptosis in several different tumor cell lines (Martinez et al., 1998; Faubion et al., 1999; Mahmoud et al., 1999; Sodeman et al., 2000). Several laboratories identified the farnesoid X receptor (FXR) as a CDCA receptor, and PKC was suggested as a signaling mediator of CDCA (Makishima et al., 1999; Parks et al., 1999; Wang et al., 1999; Rust et al., 2000; Song et al., 2001).

Various activities of CDCA on cells have also been reported. Hydrophobic CDCA is known as a tumor promoter in certain experimental systems. It also enhances cell transformation in vitro and apoptosis in several different tumor cell lines, such as HCT116 and HT29 human colon cancer cells (Brady et al., 1996; Martinez et al., 1998). During the apoptotic process of HCT116, the caspase-3 activity was stimulated, and the caspase- 3 activity and apoptosis could be suppressed by $b c l-2$. Noticeably, the over-expression of $b c l-2$ or the inhibition of PKC activity caused the mode of cell death to switch from apoptosis to necrosis (LaRue et al., 2000). CDCA also induced AP-1 activity in human colon adenocarcinoma cells (Hirano et al., 1996; Glinghammar et al., 1999) and in hepatic stellate cells (Brady et al., 1996). In human esophageal adenocarcinoma cells, CDCA induced the cyclooxygenase-2 expression (Zhang et al., 1998). In human colon cancer Caco-2 cells, it induced the $c$-fos gene expression through PKC activation (Di Toro et al., 2000). The ability of bile acids to induce apoptosis, as well as stimulate proto-oncogenes such as cyclooxygenase-2, c-myc and AP-1, suggests that bile acids may exert their tumor-promoting activity by modulating intracellular signaling and gene expression. This will consequently change cell growth and tumorigenesis (Zhang et al., 1998; Qiao et al., 2001a; Debruyne et al., 2001).

Glycochenodeoxycholic acid (GCDC), a toxic, dihydroxy, and hydrophobic bile acid, induced Fas oligomerization and activated caspase-8, which resulted in apoptosis, independently of Fas ligand (Faubion et al., 1999). The taurine conjugate of CDCA, taurochenodeoxycholate (TCDC), activates the phosphatidylinositol 3-kinase (PI3K), a potent activator of survival signals. This raises the possibility that nontoxic and hydrophobic bile acids do not trigger apoptosis, because they activate a PI3K-dependent survival signaling pathway (Yao and Cooper, 1995; Misra et al., 1998; Rust et al., 2000). TCDC also activated NF- $\mathrm{KB}$ in a PI3K- and
PKC $\xi$ - dependent manner. This process could contribute to the reduction of cytotoxicity by TCDC (Rust et al., 2000).

Secondary bile acid, DCA, had a similar activity in apoptosis induction with a CDCA (Martinez et al., 1998; Zhang et al., 1998; Glinghammar et al., 1999; Di Toro et al., 2000). DCA induced DNA damage and apoptosis in human colon epithelial cells in a p53-independent manner (Powolny et al., 2001). Also, DCA could suppress p53 by stimulating the proteosome-mediated p53 protein degradation (Qiao et al., 2001a). Interestingly, apoptosis induction by DCA could be suppressed by inhibiting PKC activity with calphostin C (Martinez et al., 1998), and also be modulated by the extracellular signal-regulated kinase (ERK) (Qiao et al., 2001a). DCA also caused ligand-independent activation of both the epidermal growth factor receptor (EGFR) and FAS receptor in primary hepatocytes via the MAPK signaling pathway (Qiao et al., 2001b).

Glycodeoxycholic acid (GDCA) induced apoptosis in hepatocytes by a mechanism that is associated with DNA cleavage by endonucleases (Kwo et al., 1995), and induction of the TRAIL-receptor 2/DR5 expression (Higuchi et al., 2001). In addition to this, the overexpression of $\mathrm{PKC} \xi$ blocked the GCDA-induced apoptosis (Rust et al., 2000).

LCA, hydrophobic secondary bile acid, induced AP-1 activity in both HT29 and HCT116 (Glinghammar et al., 1999), and induced egr in hepatic stellate cells with the activation of MAPK (Brady et al., 1996). LCA also induced the $c$-fos gene expression through PKC in human colon cancer Caco-2 cells (Di Toro et al., 2000).

A representative secondary bile acid, UDCA, is known as a chemopreventive agent (Earnest et al., 1994) by inhibiting cell proliferation without inducing apoptosis (Martinez et al., 1998). UDCA inhibited progression through the cell cycle, and caused cells to become blocked in the G1 phase of the cell cycle (Martinez et al., 1998). UDCA also prevented bilirubininduced apoptosis that is aggravated by CDCA in cultured rat neural cells (Silva et al., 2001). Hydrophilic UDCA and its taurine and glycine conjugates protect cells against apoptosis that is induced by several hydrophobic bile acids (Heuman et al., 1991; Heuman et al., 1994). This protective effect is due to the direct prevention of mitochondrial membrane perturbation (Rodrigues et al., 1998).

\section{Cellular and biochemical effects of synthetic bile acids}

Figure 1 shows a graphical representation of the CDCA and UDCA structures and where modifications occurred. A new glycine methyl ester conjugate of UDCA, HS-1030, induced apoptosis in HepG2 human hepatocellular carcinoma cells and MCF-7 human breast carcinoma cells (Baek et al., 1997; Park et al., 1997; Im et al., 1999). It had little cytotoxic effect in the immortalized human breast epithelial cell line MCF10A (Im, E.O. \& Kim, N.D., unpublished data). HS-1030 also showed inhibitory effects on embryonic angiogenesis in chick 
<smiles>[R]C(=O)CCC(C)C1CCC2C3C(CCC12C)C1(C)CC[C@@H](O)CC1C[C@H]3O</smiles>

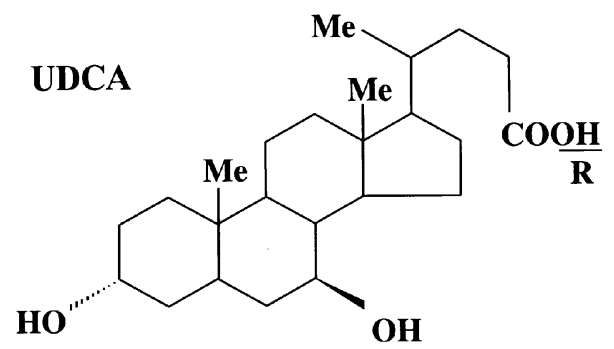

Fig. 1. Structures of UDCA and CDCA. New synthetic bile acids were made by modification at the side-chain labeled $\mathrm{R}$.

embryo chorioallantoic membranes that showed antiangiogenic activity, and exhibited no cytotoxic effect on the calf pulmonary artery endothelial (CPAE) cells (Suh et al., 1997). Additionally, HS-1030 and L-phenylalanine benzyl ester conjugate of UDCA, HS-1183, inhibited SV40 DNA replication, and predominantly inhibited the initiation stage of DNA relication (Kim et al., 1999). The L-phenyl alanine benzyl ester conjugate (HS-1199), the $\beta$-alanine benzyl ester conjugate (HS-1200) of CDCA, and HS-1183 induced apoptosis via a p53-independent pathway in human breast carcinoma cells (Im et al., 2001). HS-1199 and HS-1200 also showed apoptotic activity in human leukemic T cells through the activation of caspases (Choi et al., 2001).

Cell cycle arrest Synthetic bile acids play a novel role in regulating the cell cycle. In mammalian cells, D-type cyclins are synthesized during the G1 phase. They regulate G1/S transition with their partners, cdks (Nigg, 1995; Pines, 1995; Sherr and Roberts, 1995). The cdk inhibitors regulate cellcycle progression by association with cyclin/cdk complexes (Fernandez et al., 1998; Hui et al., 1998). The results from the immunoblotting analysis demonstrated that HS-1199 and/or HS-1200 selectively down-regulate the intracellular protein levels of D-type cyclins, which play essential roles as positive regulators of cdk4 and cdk6 activities. There was no change in the levels of cdks (Im et al., 2001).

Moreover, the synthetic bile acids selectively induced the expression of the cdk inhibitor p21 $1^{\text {WAFI/CIPI }}$ without affecting the protein level of $\mathrm{p} 53$. They also increase the $\mathrm{p} 21^{\text {WAFI/CIPI }}$ protein that is tightly associated with cdk2 (Im et al., 2001). These results demonstrated that the down-regulations of cdks and cyclin E-dependent kinase activities were mainly caused by the selective induction of the $\mathrm{p} 21^{\text {WAFI/CIPI }}$ expression. The effect of HS-1199 and HS-1200 was more distinct than that of
HS-1183. Furthermore, the increased $\mathrm{p} 21^{\text {WAFI/CIPI }}$ protein by treatment with the synthetic bile acid derivatives was strongly associated with PCNA, which is required for the process of DNA synthesis by DNA polymerases (Li et al., 1994; Gibbs et al., 1997). These results suggest that the synthetic bile acidinduced $\mathrm{p} 21^{\text {WAFI/CIPI }}$ could inhibit the PCNA function by direct binding to PCNA.

Apoptosis The growth inhibitory effect of the synthetic bile acids was derived from the induction of apoptosis with several characteristic features. After exposure to synthetic bile acids, morphological changes in the nuclei were observed by nuclear staining with DAPI (Im et al., 1999). As shown by a flow cytometry analysis, the sub G1 population of cells was increased by synthetic bile acids.

Apoptotic cell death by the synthetic bile acid derivatives was also confirmed by DNA ladder formation (Im et al., 2001). It has been proposed that DNA fragmentation resulted from the loss of compartmentalization of DNase I, which would reach the nucleus due to the breakdown of the endoplasmic reticulum and the nuclear membrane (Fraser et al., 1996). This cleavage produces ladders of DNA fragments that are the size of integer multiples of a nucleosome length (180-200 bp) (Huang et al., 1997).

When cells undergo apoptosis, specific degradation of several proteins (such as lamin B and poly (ADP-ribose) polymerase (PARP), followed by internucleosomal DNA degradation) has been reported (Berger, 1985; Neamati et al., 1995). PARP is an enzyme that is involved in DNA repair and genomic integrity, and used as a biochemical marker of chemotherapy-induced apoptosis, as mentioned previously (Cohen, 1997; Rosen and Casciola-Rosen, 1997; Konopleva et al., 1999). A collapse of chromatin and nuclear structure in apoptosis is also consistent with the degradation of lamins, which are a part of the nuclear envelope (Oberhammer et al., 1994). Cleavages of PARP and lamin B were shown by the treatment of the synthetic bile acid derivatives (Im et al., 2001).

Several members of the caspase family, including caspase- 3 and 8 , have been identified as proteases, which cleave the PARP protein (Lazebnik et al., 1994). Therefore, subsequent experiments addressed the issue of whether or not synthetic bile acid derivative-induced apoptosis was associated with caspase activation. The immunoblot analysis revealed that the steady-state levels of both procaspase- 3 and procaspase- 8 proteins were markedly decreased in cells that were treated with the synthetic bile acids (Choi et al., 2001). This suggests that the caspase-mediated signaling might contribute to the synthetic bile acid-mediated apoptosis in Jurkat cells. By comparison, CDCA and UDCA themselves did not affect the levels of caspase- 3 or caspase- 8 expressions. Furthermore, the apoptotic markers (including chromatin condensation, DNA ladder formation, and proteolytic cleavage of PARP) were completely prevented when the cells were pretreated with zVAD-fmk and DEVD-fmk, which is a broad-spectrum 
inhibitor of caspases and a specific inhibitor of caspase-3 (Choi et al., 2001), respectively.

The Bcl-2 oncoprotein, and other related proteins, might play an important role in determining whether cells undergo apoptosis. The increased expression of Bax can induce apoptosis by suppressing the activity of Bcl-2 (Findley et al., 1997). It was also reported that the ratio of Bcl-2 to Bax, rather than Bcl-2 alone, was more important for the survival of drug-induced apoptosis (Salomons et al., 1997). Although the Bcl-2 expression was not significantly changed in MCF-7 cells that were treated with the synthetic bile acid derivatives, there was a significant increase of the Bax expression (Im et al., 2001). Additionally, the expression level of Bax was increased and that of Bcl-2 was markedly decreased in MDAMB-231 cells (Im et al., 2001). Therefore, the increased ratio of $\mathrm{Bax}$ to $\mathrm{Bcl}-2$ might contribute to the initiation of apoptosis in the synthetic bile acid-treated cells.

In apoptosis, cytosolic $\mathrm{Ca}^{2+}$ affects the mitochondrial permeability transition pore complex directly, and induces the rupture of the outer membrane of mitochondria (Bernardi and Petronilli, 1996). The elevation of $\mathrm{Ca}^{2+}$ also induces the release of caspase-activating proteins ( $\mathrm{Li}$ et al., 2000). $\mathrm{A} \mathrm{Ca}^{2+}$ chelator, BAPTA/AM, was pre-treated to cells before the HS$1200(25 \mu \mathrm{M})$ treatment in MCF-7 cells (Im, E.O. and Kim, N.D., unpublished data). However, the BAPTA/AM cotreatment could not block the synthetic bile acid derivatives induced apoptosis, and it also slightly potentiated apoptosis induction. Therefore, $\mathrm{Ca}^{2+}$ did not act as a signaling molecule in the synthetic bile acid-induced apoptosis.

\begin{abstract}
Alterations in mitochondrial function Alterations in the mitochondrial function in general, and induction of the mitochondrial permeability transition (MPT), play a key part in the regulation of apoptosis (Kroemer et al., 1997; Green and Reed, 1998; Susin et al., 1999). Moreover, antioxidants and blockers of the mitochondrial permeability transition inhibited the hydrophobic bile acid-induced rat hepatocyte apoptosis (Yerushalmi et al., 2001). The mitochondrial membrane potential $(\Delta \Psi \mathrm{m})$ can be monitored in living cells using numerous potentially sensitive dyes. With the use of these dyes, the $\Delta \Psi \mathrm{m}$ dissipates before the plasma membrane disrupts, not only in apoptosis but also in numerous in vitro models of necrosis (Kroemer et al., 1998). Synthetic bile acid derivatives induced loss of $\Delta \Psi \mathrm{m}$ in human retinal pigment epithelial cells (Yoon et al., 2001).
\end{abstract}

\section{Mechanism of action of synthetic bile acid in apoptosis}

Although the detailed molecular mechanism of the induction of apoptosis by the synthetic bile acids is still unknown, it can be speculated that the possible mechanism is based on the data of previous studies with natural bile acids (Fig. 2). Bile salt cytotoxicity, both in vivo and in vitro, was mediated by the death receptor Fas (Faubion et al., 1999; Miyoshi et al., 1999).

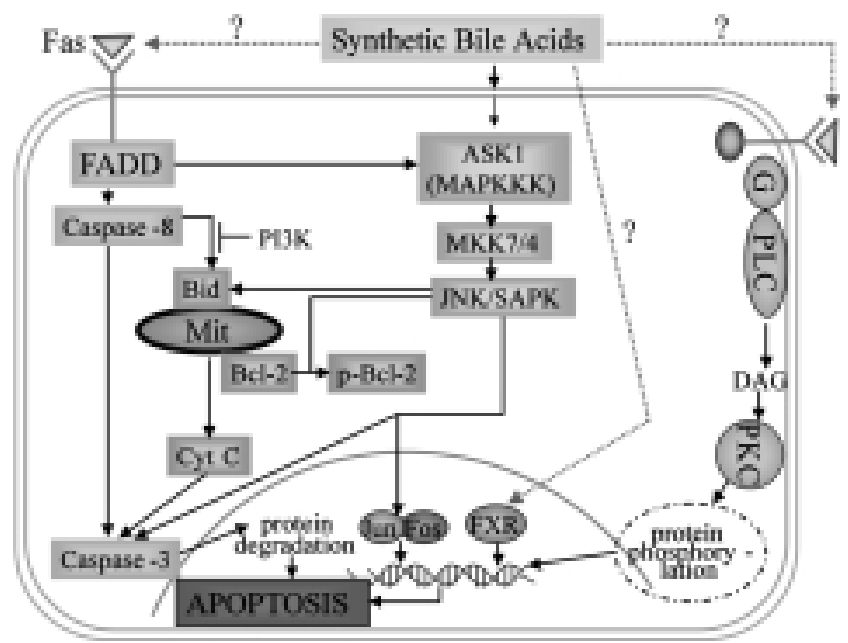

Fig. 2. A scheme depicting the proposed role of the synthetic bile acids as mediators of apoptosis. The synthetic bile acids may induce apoptosis by direct binding to Fas receptors, caspase8 activation, and Bid translocation to mitochondria or via the PKC-dependent signaling pathway. Another possible mechanism is that the synthetic bile acids may bind to FXR and exert their actions. The dotted arrows represent steps that might be involved in apoptosis by the synthetic bile acids. ASK, apoptosis signaling kinase; Cyt c, cytochrome c; DAG, diacylglycerol; FADD, Fasassociated death domain; G, G-protein; JNK, c-Jun $\mathrm{NH}_{2}$-terminal kinase; MAPKKK, mitogen activated protein kinase; Mit, mitochondria; MKK, MAP kinase; PLC, phospholipase C.

GCDC induced Fas oligomerization and activated caspase-8, resulting in apoptosis, independently of Fas ligand (Faubion et al., 1999) in rodent hepatocyte. Another study demonstrated that enhanced PI3K activity inhibited the Fas-mediated apoptosis (Cristofano et al., 1999). Thus, the bile acid-induced Fas activation appears to be inhibited by the simultaneous activation of a kinase-dependent, anti-apoptotic signaling pathway that blocks the cytotoxicity of bile acids. The taurine conjugate of CDCA, TCDC, activates a PI3K survival pathway that blocks the inherent toxicity of GCDC (Rust et al., 2000). NF- $\mathrm{\kappa B}$ is one of the downstream targets of the TCDC stimulated PI $3 \mathrm{~K}$ activity. Thus, NF- $\kappa \mathrm{B}$ may suppress the bile acid-mediated Fas/caspase- 8 activation by upregulating antiapoptotic proteins, such as the inhibitor of the apoptosis protein-1 (cIAP-1) (Wang et al., 1996). Therefore, we assume that the synthetic bile acids may induce apoptosis by activating the Fas receptor-signaling pathway.

Interestingly, there have been numerous reports that the PKC signaling pathway was involved in the effect of several bile acids. Bile acids can activate PKC directly in vitro and in vivo assays (Fitzer et al., 1987; Huang et al., 1992; Wali et al., 1995; Morgan et al., 1997; Qiao et al., 2000), perhaps by substituting for phosphatidyl serine (Ward and OBrian, 1988), and indirectly by stimulating the phospholipase $\mathrm{C}$ activity. This results in the increased production of diacylglycerol, a known co-factor that is required for PKC activation (Craven $e t$ 
al., 1987). Therefore, it is possible that PKC might be a likely component of the mechanism by which the synthetic bile acids exert their biological effects.

We also presume that the synthetic bile acids, which have chemical structures that are similar to glucocorticoids, may directly affect the signaling molecules in cytosol by passing through the cell membrane. Moreover, it was recently reported that CDCA exerted its action by binding to an orphan nuclear receptor, FXR, as a natural ligand (Makishima et al., 1999; Parks et al., 1999; Wang et al., 1999; Song et al., 2001). Similarly, the synthetic CDCA derivatives may bind FXR, or even possibly be a ligand for a co-activator/co-repressor type of molecule. Further experiments are open in the future to elucidate the structure/function relationship of the synthetic CDCA and UDCA derivatives.

\section{Summary and implications}

In this paper, we outlined the current understanding of natural and synthetic bile acid signaling in apoptosis. Much insight was gained in recent years, particularly with respect to the inducers of the hydrophobic bile acid signaling cascade. Moreover, the newly synthesized bile acids also induced apoptosis in several human cancer cells, which were not derived from hepatocytes, cholangiocytes, and ileal enterocytes. Therefore, the new synthetic bile acids might be applicable novel apoptosis mediators for the treatment of various cancer cells.

In human breast and prostate cancer cells with different tumor suppressor p53 status, the synthetic bile acid derivativeinduced growth inhibition and apoptosis were associated with the up-regulation of Bax and p21 $1^{\text {WAFI/CIPI }}$, and the effects were mediated via a p53-inpendent pathway. In Jurkat human $\mathrm{T}$ cell leukemia, the synthetic bile acid derivatives induced apoptosis through caspase activation. Additionly, the synthetic bile acids induced apoptosis in a JNK dependent manner in SiHa human cervical cancer cells, PC3 prostate cancer cells, HT29 colon cancer cells, and TE671 brain tumor cells (Im, E.O., Yoo, Y.H., and Kim, N.D., unpublished data).

Alterations in cell survival play a critical role for the pathogenesis of various human diseases, including cancer (Thompson, 1995). Administration of modified bile acids that induce apoptosis might represent a rational therapy for tumors that originate from several different types of cells. Therefore, these novel derivatives of bile acids may represent promising chemical entities, which specifically target various cancer cells by triggering apoptosis. These could be important lead compounds for the development of new anticancer agents that are based on the structure of bile acids.

Acknowledgments This work was supported by grant No. R01-2001-00145 from the Korea Science \& Engineering Foundation and by a Pusan National University Research Grant.

\section{References}

Baek, J. H., Kim, J., Kang, C., Lee, Y. S. and Kim, K. W. (1997) Induction of apoptosis by bile acids in HepG2 human hepatocellular carcinoma cells. Korean J. Physiol. Pharmacol. 1, 107-115.

Berger, N. A. (1985) Poly(ADP-ribose) polymerase in the cellular response to DNA damage. Radiat. Res. 101, 4-15.

Bernardi, P. and Petronilli, V. (1996) The permeability transition pore as a mitochondrial calcium release channel: a critical appraisal. J. Bioenerg. Biomembr. 28, 131-138.

Blake J., Roberts, P. J., Faubion, W. A., Kominami, E. and Gores, G. J. (1998) Cystatin A expression reduces bile salt-induced apoptosis in a rat hepatoma cell line. Am. J. Physiol. 275, G723-G730.

Brady, L. M., Beno, D. W. A. and Davis, B. H. (1996) Bile acid stimulation of early growth response gene and mitogenactivated protein kinase is protein kinase C-dependent. Biochem. J. 316, 756-769.

Choi, Y. H., Im, E. O., Suh, H., Jin, Y., Lee, W. H., Yoo, Y. H., Kim, K. W. and Kim, N. D. (2001) Apoptotic activity of novel bile acid derivatives in human leukemic $\mathrm{T}$ cells through the activation of caspases. Int. J. Oncol. 18, 979-984.

Cohen, G. M. (1997) Caspases: the executioners of apoptosis. Biochem. J. 326, 1-16.

Craven, P. A., Pfanstiel, J. and DeRubertis, F. R. (1987) Role of activation of protein kinase $\mathrm{C}$ in the stimulation of colonic epithelial proliferation and reactive oxygen formation by bile acids. J. Clin. Invest. 79, 532-541.

Cristofano, A. D., Kotsi, P., Peng, Y. F., Cordon-Cardo, C., Elkon, K. B. and Pandolfi, P. P. (1999) Impaired Fas response and autoimmunity in Pten +/-mice. Science 285, 2122-2125.

Debruyne, P. R., Bruyneel, E. A., Li, X., Zimber, A., Gespach, C. and Mareel, M. M. (2001) The role of bile acids in carcinogenesis. Mutat. Res. 480-481, 359-369.

Di Toro, R., Campana, G., Murari, G. and Spampinato, S. (2000) Effects of specific bile acids on c-fos messenger RNA levels in human colon carcinoma Caco-2 cells. Eur. J. Pharm. Sci. 11, 291-298.

Earnest, D. L., Holubec, H., Wali, R. K., Jolley, C. S., Bissonette, M., Bhattacharyya, A. K., Roy, H., Khare, S. and Brasitus, T. A. (1994) Chemoprevention of azoxymethane-induced colonic carcinogenesis by supplemental dietary ursodeoxycholic acid. Cancer Res. 54, 5071-5074.

Faubion, W. A., Guicciardi, M. E., Miyoshi, H., Bronk, S. F., Roberts, P. J., Svingen, P. A., Kaufmann, S. H. and Gores, G. J. (1999) Toxic bile salts induce rodent hepatocyte apoptosis via direct activation of Fas. J. Clin. Invest. 103, 137-145.

Fernandez, P. L., Jares, P., Rey, M. J., Campo, E. and Cardesa, A. (1998) Cell cycle regulators and their abnormalities in breast cancer. Mol. Pathol. 51, 305-309.

Findley, H. W., Gu, L., Yeager, A. M. and Zhou, M. (1997) Expression and regulation of Bcl-2, Bcl-xl, and Bax correlate with p53 status and sensitivity to apoptosis in childhood acute lymphoblastic leukemia. Blood 89, 2986-2993.

Firtzer, C. J., O'Brian, C. A., Guillem, J. G. and Weinstein, I. B. (1987) The regulation of protein kinase C by chenodeoxycholate, deoxycholate and several structurally related bile acids. Carcinogenesis 8, 217-220. 
Fraser, M. J., Tynan, S. J., Papaioannou, A., Ireland, C. M. and Pittman, S. M. (1996) Endo-exonuclease of human leukemic cells: evidence for a role in apoptosis. J. Cell Sci. 109, 23432360.

Gibbs, E., Kelman, Z., Gulbis, J. M., O’Donnell, M., Kuriyan, J., Burgers, P. M. J. and Hurwitz, J. (1997) The influence of the proliferating cell nuclear antigen-interacting domain of p21(CIP1) on DNA synthesis catalyzed by the human and Saccharomyces cerevisiae polymerase delta holoenzymes. $J$. Biol. Chem. 272, 2373-2381.

Glinghammar, B., Holmberg, K. and Rafter, J. (1999) Effects of colonic lumenal components on AP-1-dependent gene transcription in cultured human colon carcinoma cells. Carcinogenesis 20, 969-976.

Green, D. R. and Reed, J. C. (1998) Mitochondria and apoptosis. Science 281, 1309-1312.

Heuman, D. M., Mills, A. S., McCall, J., Hylemon, P. B., Pandak, W. M. and Clahcevic, Z. R. (1991) Conjugates of ursodeoxycholate protect against cholestasis and hepatocellular necrosis caused by more hydrophobic bile salts. In vivo studies in the rat. Gastroenterology 100, 203-211.

Heuman, D. M. and Bajaj, R. (1994) Ursodeoxycholate conjugates protect against disruption of cholesterol-rich membranes by bile salts. Gastorenterology 106, 1333-1341.

Higuchi, H., Bronk, S. F., Takikawa, Y., Wernebrg, N., Takimoto, R., El-Deiry, W. and Gores, G. J. (2001) The bile acid glycochenodeoxycholate induces TRAIL-receptor 2/DR5 expression and apoptosis. J. Biol. Chem. 276, 38610-38618.

Hirano F., Tanada, H., Makino, Y., Okamoto, K., Hiramoto, M., Handa, H. and Makino, I. (1996) Induction of the transcription factor AP-1 in cultured human colon adenocarcinoma cells following exposure to bile acids. Carcinogenesis 17, 427-433.

Hofmann, A. F. (1984) Chemistry and enterohepatic circulation of bile acids Hepatology 4, (suppl.), 4S-14S.

Huang, P., Ballal, K. and Plunkett, W. (1997) Biochemical characterization of the protein activity responsible for high molecular weight DNA fragmentation during drug-induced apoptosis. Cancer Res. 57, 3407-3414.

Huang, X. P., Fan, X. T., Dexjeux, J. F. and Vastagna, M. (1992) Bile acids, non-phorbol-ester-type tumor promoters, stimulate the phosphorylation of protein kinase $\mathrm{C}$ substrates in human platelets and colon cell line HT29. Int. J. Cancer 52, 444-450.

Hui, A. M., Makuuchi, M. and Li, X. (1998) Cell cycle regulators and human hepatocarcinogenesis. Hepatogastroenterology 45, $1635-1642$.

Hunter, T. (1995) Protein kinases and phosphatases: the yin and yang of protein phosphorylation and signaling. Cell 80, 225236.

Im, E. O., Lee, S., Suh, H., Kim, K. W., Bae, Y. T. and Kim, N. D. (1999) A novel ursodeoxycholic acid derivative induces apoptosis in human MCF-7 breast cancer cells. Pharm. Pharmacol. Commun. 5, 293-298.

Im, E. O., Choi, Y. H., Paik, K. J., Suh, H., Jun, Y., Kim, K. W., Yoo, Y. H. and Kim, N. D. (2001) Novel bile acid derivatives induce apoptosis via a p53-independent pathway in human breast carcinoma cells. Cancer Lett. 163, 83-93.

Kim, D. K., Lee, J. R., Kim, A., Lee, A., Yoo, M. A., Kim, K. W., Kim, N. D. and Suh, H. (1999) Inhibition of initiation of simian virus 40 DNA replication in vitro by the ursodexocycholic acid and its derivatives. Cancer Lett. 146,
147-153.

Konopleva, M., Zhao, S., Xie, Z., Segall, H., Younes, A., Claxton, D. F., Estrov, Z., Kornblau, S. M. and Andreeff, M. (1999) Apoptosis. Molecules and mechanisms. Adv. Exp. Med. Biol. 457, 217-236.

Kroemer, G., Zamzami, N. and Susin, S. A. (1997) Mitochondrial control of apoptosis. Immunol. Today 18, 44-51.

Kroemer, G., Dallaporta, B. and Resche-Rigon, M. (1998) The mitochondrial death/life regulator in apoptosis and necrosis. Annu. Rev. Physiol. 60, 619-642.

Kwo, P., Patel, T., Bronk, S. F. and Gores, G. J. (1995) Nuclear serine protease activity contributes to bile acid-induced apoptosis in hepatocytes. Am. J. Physiol. 268, 613-621.

LaRue, J. M., Stratagoules, E. D. and Martinez, J. D. (2000) Deoxycholic acid-induced apoptosis is switched to necrosis by bcl-2 or calphostin C. Cancer Lett. 152, 107-113.

Lazebnik, Y. A., Kaufmann, S. H., Desnoyers, S., Poirier, G. G. and Earnshaw, W. C. (1994) Cleavage of poly(ADP-ribose) polymerase by a proteinase with properties like ICE. Nature 371, 346-347.

Li, R., Waga, S., Hannon, G. J., Beach, D. and Stillman, B. (1994) Differential effects by the p21 CDK inhibitor on PCNAdependent DNA replication and repair. Nature 371, 534-537.

Li, M., Kondo, T., Zhao, Q. L., Li, F. J., Tanabe, K., Arai, Y., Zhou, Z. C. and Kasuya, M. (2000) Apoptosis induced by cadmium in human lymphoma U937 cells through $\mathrm{Ca}^{2+}$-calpain and caspase-mitochondria-dependent pathways. J. Biol. Chem. 275, 39702-39709.

Mahmoud, N. N., Dannenberg, A. J., Bilinski, R. T., Mestre, J. R., Chadburn, A., Churchill, M., Martucci, C. and Bertagnolli, M. M. (1999) Administration of an unconjugated bile acid increases duodenal tumors in a murine model of familial adenomatous polyposis. Carcinogenesis 20, 299-303.

Makishima, M., Okamoto, A. Y., Repa, J. J., Tu, H., Learned, R. M., Luk, A., Hull, M. V., Lustig, K. D., Mangelsdorf, D. J. and Shan, B. (1999) Identification of a nuclear receptor for bile acids. Science 284, 1362-1365.

Marshall, C. J. (1995) Specificity of receptor tyrosine kinase signaling: transient versus sustained extracellular signalregulated kinase activation. Cell 80, 179-185.

Martinez, J. D., Stratagoules, E. D., LaRue, J. M., Powell, A. A., Gause, P. R., Craven, M. T., Payne, C. M., Powell, M. B., Gerner, E. W. and Earnest, D. L. (1998) Different bile acids exhibit distinct biological effects: the tumor promoter deoxycholic acid induces apoptosis and the chemopreventive agent ursodeoxycholic acid inhibits cell proliferation. Nutr. Cancer 31, 111-118.

Martinez-Diez, M. C., Serrano, M. A., Monte, M. J. and Marin, J. J. (2000) Comparison of the effects of bile acids on cell viability, and DNA synthesis by rat hepatocytes in primary culture. Biochim. Biophys. Acta 1500, 153-160.

Misra, S., Ujhazy, P., Gatmaitan, Z., Varticovski, L. and Arias, I. M. (1998) The role of phosphoinositide 3-kinase in taurocholate-induced trafficking of ATP-dependent canalicular transporters in rat liver. J. Biol. Chem. 273, 26638-26644.

Miyoshi, H., Rust, C., Rober, P. J., Burgart, L. J. and Gore, G. J. (1999) Hepatocyte apoptosis after bile duct ligation in the mouse involves Fas. Gastroenterology 117, 669-677.

Mohammad, R. M., Varterasian, M. L., Almatchy, V. P., Hannoudi, G. N., Pettit, G. R. and Al-Katib, A. (1998) Successful 
treatment of human chronic lymphocytic leukemia xenografts with combination biological agents auristatin PE and bryostatin 1. Clin. Cancer Res. 4, 1337-1343.

Morgan, W. A., Sharma, P., Kaler, B. and Bach, P. H. (1997) The modulation of protein kinase $\mathrm{C}$ by bile salts. Biochem. Soc. Trans. 25, 75S.

Neamati, N., Fernandez, A., Wright, S., Kiefer, J. and McConkey, D. J. (1995) Degradation of lamin B1 precedes oligonucleosomal DNA fragmentation in apoptotic thymocytes and isolated thymocyte nuclei. J. Immunol. 154, 3788-3795.

Nigg, E. A. (1995) Cyclin-dependent protein kinases: key regulators of the eukaryotic cell cycle. Bioessays 17, 471-480.

Oberhammer, F. A., Hochegger, K., Froschl, G., Tiefenbacher, R. and Pavelka, M. (1994) Chromatin condensation during apoptosis in accompanied by degradation of lamin $\mathrm{A}+\mathrm{B}$, without enhanced activation of cdc2 kinase. J. Cell Biol. 126, 827-837.

Park, Y. H., Kim, J., Baek, J., Jung, E., Kim, T., Suh, H., Park, M. H. and Kim, K. W. (1997) Induction of apoptosis in HepG2 human hepatocellular carcinoma cells by a novel derivative of ursodeoxycholic acid (UDCA). Arch. Pharm. Res. 20, 29-33.

Parks, D. J., Blanchard, S. G., Bledsoe, R. K., Chandra, G., Consler, T. G., Kliewer, S. A., Stimmel, J. B., Willson, T. M., Zavacki, A. M., Moore, D. D. and Lehmann, J. M. (1999) Bile acids: natural ligands for an orphan nuclear receptor. Science 284, 1365-1368.

Patel, T., Bronk, S. F. and Gores, G. J. (1994) Increases of intracellular magnesium promote glycodeoxycholate-induced apoptosis in rat hepatocytes. J. Clin. Invest. 94, 2183-2192.

Patel, T., Roberts, L. R., Jones, B. A. and Gores, G. J. (1998) Dysregulation of apoptosis as a mechanism of liver disease: an overview. Semin. Liver Dis. 18, 105-114.

Payne, C. M., Crowley, C., Washo-Stultz, D., Briehl, M., Bernstein, H., Bernstein, C., Beard, S., Holubec, H. and Warneke, J. (1998) The stress-response proteins poly(ADPribose) polymerase and NF- $\mathrm{KB}$ protect against bile salt-induced apoptosis. Cell Death Differ. 5, 623-636.

Pines, J. (1995) Cyclins and cyclin-dependent kinases: a biochemical view. Biochem. J. 308, 697-711.

Porsch Hallstrom, I., Svensson, D. and Blanck, A. (1991) Sexdifferentiated deoxycholic acid promotion of rat liver carcinogenesis is under pituitary control. Carcinogenesis 12, 2035-2040.

Powell, A. A., LaRue, J. M., Batta, A. K. and Martinez, J. D. (2001) Bile acid hydrophobicity is correlated with induction of apoptosis and/or growth arrest in HCT116 cells. Biochem. J. 356, 481-486.

Powolny, A., Xu, J. and Loo, G. (2001) Deoxycholate induces DNA damage and apoptosis in human colon epithelial cells expressing either mutant or wild-type p53. Int. J. Biochem. Cell. Biol. 33, 193-203.

Qiao, D., Chen, W., Stratagoules, E. D. and Martinez, J. D. (2000) Bile acid-induced activation of activator protein-1 requires both extracellular signal-regulated kinase and protein kinase C signaling. J. Biol. Chem. 275, 15090-15098.

Qiao, D., Stratagouleas, E. D. and Martinez, J. D. (2001a) Activation and role of mitogen-activated protein kinases in deoxycholic acid-induced apoptosis. Carcinogenesis 22, 35-41.

Qiao, L., Studer, E., Leach, K., McKinstry, R., Gupta, S., Decker,
R., Kukreja, R., Valerie, K., Nagarkatti, P., El Deiry, W., Molkentin, J., Schmidt-Ullrich, R., Fisher, P. B., Grant, S., Hylemon, P. B. and Dent, P. (2001b) Deoxycholic acid (DCA) causes ligand-independent activation of epidermal growth factor receptor (EGFR) and FAS receptor in primary hepatocytes: inhibition of EGFR/mitogen-activated protein kinase-signaling module enhances DCA-induced apoptosis. Mol. Biol. Cell 12, 2609-2645.

Rodrigues, C. M. P., Fan, G., Ma, X., Kren, B. T. and Steer, C. J. (1998) A novel role for ursodeoxycholic acid in inhibiting apoptosis by modulating mitochondrial membrane perturbation. J. Clin. Invest. 101, 2790-2799.

Rosen, A. and Casciola-Rosen, L. (1997) Macromolecular substrates for the ICE-like proteases during apoptosis. J. Cell Biochem. 64, 50-54.

Rust, C., Karnitz, L. M., Paya, C. V., Moscat, J., Simari, R. D. and Gores, G. J. (2000) The bile acid taurochenodeoxycholate activates a phosphatidylinositol 3-kinase-dependent survival signaling cascade. J. Biol. Chem. 275, 20210-20216.

Salomons, G. S., Brady, H. J., Verwijs-Janssen, M., Van Den Berg, J. D., Hart, A. A., Behrendt, H., Hahlen, K. and Smets, L. A. (1997) The Bax alpha: Bcl-2 ratio modulates the response to dexamethasone in leukemic cells and is highly variable in childhood acute leukemia. Int. J. Cancer 71, 959965.

Sherr, C. J. and Roberts, J. M. (1995) Inhibitors of mammalian G1 cyclin-dependent kinases. Genes Dev. 9, 1149-1163.

Silva, R. F., Rodrigues, C. M. and Brites, D. (2001) Bilirubininduced apoptosis in cultured rat neural cells is aggravated by chenodeoxycholic acid but prevented by ursodeoxycholic acid. J. Hepatol. 34, 402-408.

Sodeman, T., Bronk, S. F., Roberts, P. J., Miyoshi, H. and Gores, G. J. (2000) Bile salts mediate hepatocyte apoptosis by increasing cell surface trafficking of Fas. Am. J. Physiol. Gastrointest. Liver Physiol. 278, 992-999.

Song, C. S., Echchgadda, I., Baek, B. S., Ahn, S. C., Oh, T., Roy, A. K. and Chatterjee, B. (2001) Dehydroepiandrosterone sulfotransferase gene induction by bile Acid activated farnesoid x receptor. J. Biol. Chem. 276, 42549-42556.

Suh, H., Jung, E. J., Kim, T. H., Lee, H. Y., Park, Y. H. and Kim, K. W. (1997) Anti-angiogenic activity of ursodeoxycholic acid and its derivatives. Cancer Lett. 113, 117-122.

Susin, S. A., Lorenzo, H. K., Zamzami, N., Marzo, I., Snow, B. E., Brothers, G. M., Mangion, J., Jacotot, E., Costantini, P., Loeffler, M., Larochette, N., Goodlett, D. R., Aebersold, R., Siderovski, D. P., Penninger, J. M. and Kroemer, G. (1999) Molecular characterization of mitochondrial apoptosis-inducing factor. Nature 397, 441-446.

Takai, Y., Kaibuchi, K., Tsuda, T., Yamashita, T., Kikuchi, A., Tanimoto, T. and Hoshijima, M. (1986) Possible modes of action of growth factors and tumor promoters in the activation of the c-myc gene in Swiss 3T3 fibroblasts (in Japanese). Gan To Kagaku Ryoho 13, 798-805.

Wali, R. K., Frawley, B. P., Jr, Hartmann, S., Roy, H. K., Khare, S., Scaglione-Sewell, B. A., Earnest, D. L., Sitrin, M. D., Brasitus, T. A. and Bisonnette, M. (1995) Mechanism of action of chemoprotective usrodeoxycholate in the azoxymethane model of rat colonic carcinogenesis: potential roles of protein kinase C. Cancer Res. 55, 5257-5264.

Wang, C., Mayo, M. W. and Baldwin, A. S. Jr. (1996) TNF-and 
cancer therapy-induced apoptosis: potentiation by inhibition of NF-кB. Science 274, 784-787.

Wang, H., Chen, J., Hollister, K., Sowers, L. C. and Forman, B. M. (1999) Endogenous bile acids are ligands for the nuclear receptor FXR/BAR. Mol. Cell 3, 543-553.

Ward, N. E. and OBrian, C. A. (1988) The bile acid analog fusidic acid can replace phosphatidylserine in the activation of protein kinase $\mathrm{C}$ by 12-O-tetradecanoylphorbol-13-acetate in vitro. Carcinogenesis 9, 1451-1454.

Yao, R. and Cooper, G. M. (1995) Requirement for phosphatidylinositol-3 kinase in the prevention of apoptosis by nerve growth factor. Science 267, 2003-2006.

Yerushalmi, B., Dahl, R., Devereaux, M. W., Gumpricht, E. and Sokol, R. J. (2001) Bile acid-induced rat hepatocyte apoptosis is inhibited by antioxidants and blockers of the mitochondrial permeability transition. Hepatology 33, 616-626.

Yoon, H. S., Rho, J. H., Yoo, K. W., Park, W. C., Rho, S. H., Choi, Y. H., Suh, H., Kim, N. D., Yoo, K. S. and Yoo, Y. H. (2001) Synthetic bile acid derivatives induce nonapoptotic death of human retinal pigment epithelial cells. Curr. Eye Res. 22, 367-374.

Zhang, F., Subbaramaiah, K., Altorki, N. and Dannenberg, A. J. (1998) Dihydroxy bile acid activate the transcription of cycloxygenase-2. J. Biol. Chem. 273, 2424-2428.

Zheng, Z., Bernstein, H., Bernstein, C., Payne, C. M., Martinez, J. D. and Gerner, E. W. (1996) Bile acid activation of the gadd153 promoter and of p53-independent apoptosis: relevance to colon cancer. Cell Death Differ. 3, 407-414. 